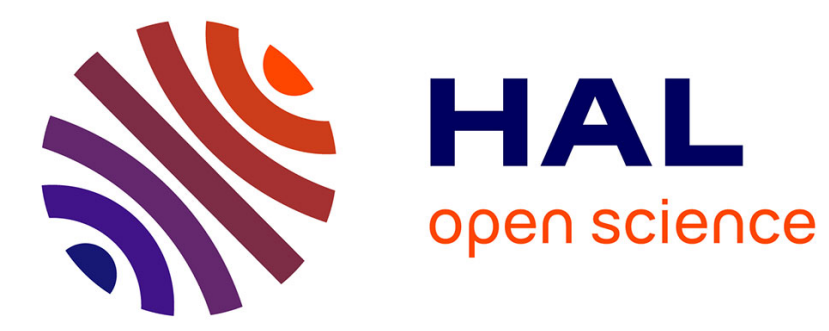

\title{
Philosophie de l'histoire et système des valeurs chez Heinrich Rickert
}

\author{
Julien Farges
}

\section{To cite this version:}

Julien Farges. Philosophie de l'histoire et système des valeurs chez Heinrich Rickert. Les études philosophiques, 2010, Rickert et la question de l'histoire, 92 (2010-1), p. 25-44. 10.3917/leph.101.0025 . halshs-01334897

\section{HAL Id: halshs-01334897 \\ https://shs.hal.science/halshs-01334897}

Submitted on 21 Jun 2016

HAL is a multi-disciplinary open access archive for the deposit and dissemination of scientific research documents, whether they are published or not. The documents may come from teaching and research institutions in France or abroad, or from public or private research centers.
L'archive ouverte pluridisciplinaire HAL, est destinée au dépôt et à la diffusion de documents scientifiques de niveau recherche, publiés ou non, émanant des établissements d'enseignement et de recherche français ou étrangers, des laboratoires publics ou privés.

\section{()ㅜ) $\Theta$}

Distributed under a Creative Commons Attribution - NoDerivatives| 4.0 International 


\section{JULIEN FARGES}

Doctorant à l'Université Paris-Sorbonne (Paris 4)

Chercheur associé aux Archives Husserl de Paris (UMR 8547, CNRS/ENS)

\section{PHILOSOPHIE DE L'HISTOIRE ET SYSTEME DES VALEURS CHEZ HEINRICH RICKERT}

Résumé : Ce travail se propose de montrer que l'intérêt de la philosophie des valeurs de Rickert relativement au champ historique ne se limite pas, comme on le croit souvent, au développement d'une logique de la connaissance historique, mais qu'au-delà de cette dimension épistémologique, elle contribue à déterminer à nouveaux frais l'idée d'une philosophie de l'histoire. Il s'agit dès lors d'élucider la thèse paradoxale selon laquelle c'est de l'édification d'un système des valeurs ineffectives que dépend pour Rickert la possibilité d'une philosophie de cette effectivité asystématique qu'est la matière historique. Il apparaît ainsi que l'idée d'un système des valeurs permet de différencier plusieurs niveaux de cette philosophie de l'histoire, qui sont autant de moments d'une critique radicale de l'historicisme.

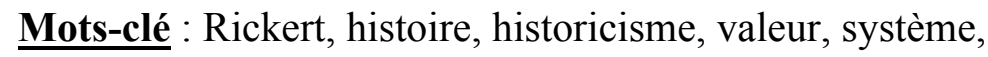

Abstract : This paper intends to show that Rickert's philosophy of values provides not only a logic of historical knowledge but also a renewed philosophy of history itself. Rickert's paradoxical claim is in fact that only a system of non effective values can allow the development of a critical philosophy of effective and non systematic history. This fundamental assertion is explained by showing how a system of values allows to divide the very idea of a philosophy of history into different levels, which are at the same time different steps on the way to a radical refutation of historicism.

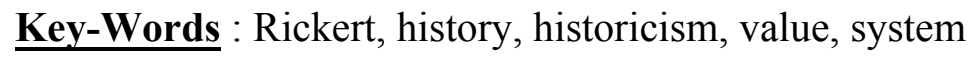


Il est particulièrement tentant de considérer l'œuvre de Rickert comme partagée en deux versants problématiques formant deux continents bien distincts, chacun étant représenté par un ouvrage fondamental inlassablement réédité (et souvent augmenté lors de chaque réédition). Le premier versant est celui de la théorie de la connaissance, représentée par L'Objet de la connaissance (ouvrage qui connut six éditions entre 1892 et 1928, au cours desquelles son ampleur fut quasiment multipliée par six $)^{1}$; le second celui de la théorie de la science, dont l'ouvrage emblématique est Les Limites de la formation de concepts dans les sciences de la nature (cinq éditions entre 1896 et 1929)². L'unité de l'ensemble reposant sur la notion de valeur, centrale de part et d'autre, l'articulation de ces deux versants peut dès lors être envisagée dans les deux sens : du premier au second comme passage de la philosophie première (qui dégage la sphère de la validité dans sa pureté et sa transcendance) à la philosophie seconde (qui exploite les ressources de la notion de valeur dans la perspective d'une fondation différenciée des sciences de la nature et des sciences de l'esprit), ou du second au premier comme introduction à la philosophie pure des valeurs à partir des problèmes épistémologiques.

Il ne s'agit évidemment pas pour nous de contester la validité de cette distinction - qui correspond à une différence cardinale dans la systématique des tâches de l'idéalisme transcendantal rickertien - mais simplement de remarquer qu'elle ne va pas sans donner de la pensée de Rickert une image trompeuse, à deux égards au moins. Une telle présentation a tout d'abord l'inconvénient de ne pouvoir rendre compte de façon satisfaisante de la signification de certains développements remarquables de la philosophie rickertienne des valeurs, qu'il s'agisse de l'effort d'unification systématique dont témoigne en particulier l'important ouvrage de 1921 intitulé le Système de la philosophie ${ }^{3}$, dont la signification demeure problématique tant qu'on en reste à cette présentation dualiste, ou qu'il s'agisse de l'infléchissement ontologique qui caractérise la dernière phase de la pensée de Rickert, laquelle s'ouvre précisément après les dernières rééditions des deux ouvrages mentionnés et donne lieu à des ouvrages comme La Logique du prédicat et le problème de l'ontologie (1930) ou Les problèmes fondamentaux de la philosophie $(1934)^{4}$. Mais surtout, et c'est le

\footnotetext{
${ }^{1}$ H. RiCKerT, Der Gegenstand der Erkenntnis. Ein Beitrag zum Problem der philosophischen Transzendenz, Tübingen, J. C. B. Mohr (Paul Siebeck), ${ }^{1} 1892,{ }^{2} 1904,{ }^{3} 1915,{ }^{4-5} 1921,{ }^{6} 1928$.

${ }^{2}$ H. RICKERT, Die Grenzen der naturwissenschaftlichen Begriffsbildung. Eine logische Einleitung in die historischen Wissenschaften, Freiburg, J. C. B. Mohr (Paul Siebeck), ${ }^{1} 1896-1902,{ }^{2} 1913,{ }^{3-4} 1921,{ }^{5} 1929$.

${ }^{3}$ H. RicKerT, System der Philosophie. Erster Teil : Allgemeine Grundlegung der Philosophie, Tübingen, J. C. B. Mohr (Paul Siebeck), 1921. Seule cette première partie sera finalement publiée par Rickert.

4 H. RiCKerT, Die Logik des Prädikats und das Problem der Ontologie, Heidelberg, Carl Winters Universitätsbuchhandlung, 1930 ; Grundprobleme der Philosophie. Methodologie, Ontologie, Anthropologie, Tübingen, J. C. B. Mohr (Paul Siebeck), 1934.
} 
point qui retiendra ici notre attention, une distinction trop appuyée entre les versants épistémologique et gnoséologique ne nous semble pas permettre de rendre compte dans son intégralité du traitement rickertien du problème de l'histoire ni de la conception qu'il développe d'une philosophie de l'histoire dans le contexte de son transcendantalisme axiologique.

En effet, du point de vue de la distinction dont nous sommes parti, la thématique de l'histoire semble se situer exclusivement du côté de la théorie de la science, où son concept reçoit dès lors une double caractérisation: l'histoire y signifie d'abord «non pas les événements eux-mêmes mais leur exposition, autrement dit la science historique ${ }^{5}$; par suite, en tant qu'historia rerum gestarum, l'histoire n'est ici que le concept logico-formel qui s'oppose au concept logique de nature dans le cadre de la distinction rickertienne entre méthode généralisante et méthode individualisante ${ }^{6}-$ elle est donc le concept méthodologique générique qui subsume toutes les sciences dont le but n'est pas la production de lois par généralisation ${ }^{7}$. Mais il est bien évident, et Rickert en est le premier convaincu, que cette thématisation logique de l'histoire est loin d'épuiser le traitement philosophique dont sa notion est susceptible et qu'elle requiert même avec une urgence particulière à une époque où le renouveau des études historiques en Allemagne au cours du $\mathrm{XIX}^{\mathrm{e}}$ siècle ne semble plus persister que sous la forme de mises en questions historicistes de l'universalité et de la rationalité scientifiques. Reste que l'évidence de la tâche d'une philosophie de l'histoire (et non plus d'une simple logique) est loin d'impliquer avec évidence la forme que doit prendre sa résolution. Celle-ci semble butter au contraire sur une difficulté de nature architectonique : car si, à coup sûr, la théorie de la science n'est pas le lieu d'une telle philosophie de l'histoire, on voit mal comment la théorie de la connaissance pourrait l'être, a fortiori si elle est en son foyer une doctrine des valeurs transcendantes dont la validité est conçue par opposition à toute effectivité ontologique ${ }^{8}$. Et pourtant, Rickert ne laisse subsister aucun doute sur ce point : dans différents textes des années 20, il s'attache à montrer qu'aucune philosophie de l'histoire n'est envisageable indépendamment de l'édification de ce qu'il appelle un système

\footnotetext{
5 H. RICKERT, Die Probleme der Geschichtsphilosophie. Eine Einleitung, Heidelberg, Carl Winters Universitätsbuchhandlung, ${ }^{3} 1924$, p. 5 ; trad. fr. par B. Hébert, Les problèmes de la philosophie de l'histoire. Une introduction, Toulouse, Presses Universitaires du Mirail, 1998, p. 55 (trad. modifiée).

${ }^{6}$ Opposition que nous préciserons d'un mot au début de notre deuxième section.

${ }^{7}$ Cf. H. RicKeRT, Science de la culture et science de la nature, trad. fr. par A.-H. Nicolas, Paris, Gallimard, 1997, p. 38 : «C'est bien plutôt un concept lui aussi logique qui pourra être opposé au concept logique de la nature comprise comme l'existence des choses dans la mesure où celle-ci est déterminée par des lois générales. Ce concept est selon moi le concept d'histoire, en son sens formel le plus étendu [...] ».

${ }^{8}$ Sur cette opposition fondamentale de la validité et de l'effectivité, nous nous permettons de renvoyer à notre « Présentation », in H. Rickert, Le système des valeurs et autres articles, Paris, Vrin, 2007, p. 22 sq.
} 
des valeurs. Sa corrélation nécessaire à un tel système montre ainsi que la philosophie de l'histoire se situe pour Rickert à la jonction de la théorie de la science et de la théorie de la connaissance. Et l'on voit par suite qu'il est possible d'user en retour de la question de l'histoire et de la définition d'une philosophie de l'histoire comme d'une pierre de touche de l'unité systématique de la philosophie de Rickert.

Mais cela suppose naturellement que soient clarifiés la signification et le rôle d'un système des valeurs relativement à la philosophie de l'histoire en général. Or il faut bien admettre que l'idée même d'une corrélation entre philosophie de l'histoire et système des valeurs est loin d'aller de soi. Remarquons en effet que du point de vue des critiques historicistes de l'universalisme philosophique, la seule affirmation d'une telle corrélation peut aisément passer pour une provocation rationaliste : l'expression de « système des valeurs » ne rassemble-t-elle pas des déterminations qui sont par principe les plus étrangères à la réalité historique : l'ineffectivité et la systématicité ? Comment pourrait-elle permettre de saisir quoi que ce soit de l'histoire effective ? Comment pourrait-on, par son moyen, édifier autre chose, sous le titre de "philosophie de l'histoire », qu'une nouvelle construction spéculative dans laquelle c'est précisément la richesse concrète de l'histoire qui est perdue ?

Le but que nous nous fixons ici est l'élucidation de cette thèse de Rickert selon laquelle une systématisation de l'ineffectif est la condition de toute philosophie de cette effectivité asystématique qu'est l'histoire. En tentant de comprendre dans quelle mesure un système des valeurs ineffectives peut bien relever du programme d'une philosophie de l'histoire, nous espérons nous donner les moyens de saisir comment philosophie de l'histoire et unification systématique du criticisme axiologique se rendent mutuellement possibles. Outre cet enjeu qui concerne l'unité de la pensée de Rickert par-delà des clivages sans doute trop schématiques, notre interrogation comporte un enjeu plus global, qui concerne la spécificité de la critique rickertienne de l'historicisme, pour autant qu'elle s'effectue au sein d'une philosophie dont le postulat de départ est que c'est la sphère culturelle et historique qui constitue « le véritable champ des décisions » philosophiques, pour user ici d'une expression husserlienne ${ }^{9}$. En d'autres termes, il s'agit de comprendre ici dans quelle mesure c'est précisément son caractère de "philosophie idéaliste orientée sur l'histoire $~^{10}$ qui fait de la philosophie rickertienne des valeurs une critique radicale de l'historicisme, et quelle est la forme propre à cette critique.

\footnotetext{
${ }^{9}$ E. HuSSERL, La crise des sciences européennes et la phénoménologie transcendantale, trad. fr. par G. Granel, Paris, Gallimard, 1976, § 58, p. 235.

${ }^{10}$ H. RickerT, Die Grenzen..., op. cit., ${ }^{1} 1902$, p. IV (nous soulignons), cité par M. FERRARI, Retours à Kant. Introduction au néo-kantisme, trad. fr. par T. Loisel, Paris, Cerf, 2001, p. 127.
} 
Notre démarche s'articulera dès lors en trois moments. Nous partirons de l'idée même d'un système des valeurs, telle qu'elle est présentée par Rickert dès 1913 dans le contexte théorique d'une philosophie pure des valeurs, pour ressaisir en quoi un tel système signifie en lui-même une critique de l'historicisme. Nous suivrons ensuite Rickert dans son ascension en deux étapes vers un sens de plus en plus profond de la philosophie de l'histoire : nous verrons comment un tel système contribue à la définition du programme théorique de chacun des deux sens de la philosophie de l'histoire, et nous tenterons à chaque fois de ressaisir, à partir de cette corrélation entre système des valeurs et philosophie de l'histoire, à la fois l'unité du criticisme axiologique de Rickert et la spécificité de son anti-historicisme.

Penchons-nous donc tout d'abord sur l'article intitulé «Le système des valeurs », publié dans la revue Logos en $1913^{11}$, et précisons d'emblée que son projet se situe dans la lignée des recherches de théorie de la connaissance menées dans L'objet de la connaissance. Pour caractériser en quelques mots l'apport essentiel de ce dernier ouvrage, on peut dire qu'il propose une fondation transcendantale de la connaissance par le dégagement, au fil d'une théorie du jugement qui distingue entre contenu jugé et acte judicatif d'évaluation pratique de ce contenu, d'une sphère de valeurs ineffectives en tant que pures exigences de validité, et qui, en tant que valeurs transcendantes, sont le véritable « objet de la connaissance » (au sens de Gegenstand) dans la mesure où elles en fondent la validité objective (la capacité à dire effectivement quelque chose d'un Objekt). Ces analyses débouchent donc non seulement sur la possibilité mais sur la nécessité d'une étude de cette sphère des valeurs transcendantes considérée en elle-même, dans son irréalité propre, et comme indépendamment de sa fonction de fondation transcendantale, que cette dernière soit objective (logique) ou subjective

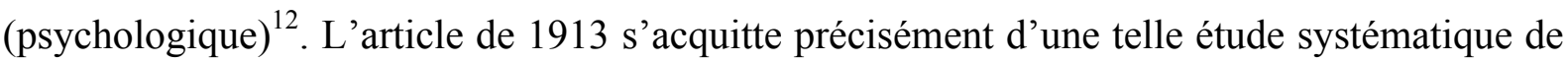
la sphère des valeurs au service d'un approfondissement de la théorie transcendantale de la connaissance. Cela posé, il faut noter que le contexte immédiat auquel Rickert rattache explicitement les recherches menées dans cet article n'est pas tant formé par ces acquis de la

\footnotetext{
${ }^{11}$ Cet article est traduit par nos soins dans le recueil auquel il donne son titre : cf. H. RICKERT, Le système des valeurs et autres articles, op. cit., p. 133-171.

${ }^{12}$ Sur cette dualité, nous renvoyons évidemment à l'article de RICKERT «Zwei Wege der Erkenntnistheorie. Transsendentalpsychologie und Transsendentallogik», in Kantstudien, XIV, 1909, p. 169 sq.; trad. fr. par A . Dewalque, Les deux voies de la théorie de la connaissance. 1909, Paris, Vrin, 2006, p. 111-162.
} 
théorie de la connaissance que par la polémique des philosophies historicistes et relativistes qui, dans la lignée de Nietzsche, dénoncent la volonté de système elle-même comme théoriquement et moralement étrangère à l'esprit de la philosophie véritable ${ }^{13}$. L'édification d'un système des valeurs doit donc contenir des ressources critiques à l'encontre de la thèse historiciste selon laquelle la prise en compte de l'histoire, dans sa diversité et sa relativité, interdit par elle-même toute systématicité. C'est cette portée critique de l'idée même d'un système des valeurs que nous voudrions souligner ici, bien qu'il soit d'ores et déjà acquis que répondre aux objections de l'historicisme par une défense et illustration de l'exigence de systématicité en philosophie ne suffit en rien à réfuter l'historicisme comme posture philosophique.

En premier lieu, on peut dire que c'est paradoxalement parce qu'il est un système des valeurs ineffectives et supra-historiques que ce système ne peut s'édifier que moyennant une prise en considération de l'effectivité historique. En effet, et c'est là un point décisif de la philosophie rickertienne des valeurs, la reconnaissance de la transcendance idéale des valeurs ne s'accompagne pas de l'affirmation d'une possible thématisation directe ou immédiate de celles-ci. Au contraire, Rickert écrit fort clairement :

Quelle que puisse bien être la fermeté de notre conviction que les valeurs valent indépendamment de nous et qu'elles confèrent à notre existence un sens « objectif », elles n'en restent pas moins accessibles à notre connaissance dans la mesure, et dans cette mesure seulement, où elles sont attachées à des biens effectifs, lesquels se présentent toujours à nous en tant que produits d'une évolution historique ${ }^{14}$.

Ce texte invite à penser qu'une philosophie des valeurs ineffectives doit nécessairement procéder d'une prise en considération du monde historique, dont les valeurs sont alors tirées par une abstraction spécifique qui ne les dégage pas comme des généralités génériques abstraites mais comme des généralités axiologiques concrètes ${ }^{15}$. Seule l'effectivité historique peut constituer le point de départ d'une philosophie systématique des valeurs, ainsi que Rickert l'affirme avec force en 1910 : « ce n'est qu'en passant par l'historique que le chemin peut mener au supra-historique. C'est donc au contact du matériau historique que la philosophie doit porter à sa propre conscience les valeurs en tant que valeurs ${ }^{16}$. Or cette déclaration de principe selon laquelle la philosophie des valeurs ineffectives n'est pas édifiée

\footnotetext{
${ }^{13}$ Cf. H. RICKERT, « Le système des valeurs », trad. cit., p. 133-134.

${ }^{14}$ Ibid., p. 134.

${ }^{15}$ Cf. par exemple sur ce point H. RICKERT, Les problèmes de la philosophie de l'histoire, op. cit., p. 49 : « On ne doit pas considérer les valeurs dans leur seule généralité abstraite [...]. Certes, la philosophie a pour objet les seules valeurs "universelles". Mais elle doit se garder de confondre cette validité avec l'universalité générique abstraite d'un contenu axiologique ».

${ }^{16}$ H. RICKERT, «Le concept de la philosophie » trad. fr. par nos soins in Le système des valeurs et autres articles, op. cit., p. 71.
} 
sur le déni du monde historique se traduit concrètement dans la méthode d'édification du système des valeurs, d'une façon que nous allons indiquer brièvement dans la mesure où peut se préciser ainsi le sens en lequel une critique de l'historicisme peut s'y alimenter.

Que les valeurs ne puissent pas être saisies autrement qu'à même les biens auxquels elles sont attachées et dont il faut les isoler par une abstraction sui generis signifie d'abord qu'un ordonnancement systématique des valeurs ne peut pas se contenter d'exposer la seule hiérarchie « des degrés de réalisation effective des valeurs ${ }^{17}$, car celle-ci peut être obtenue indépendamment de tout rattachement à des biens : la seule considération de la téléologie interne à toute réalisation effective d'une valeur quelle qu'elle soit permet en effet de distinguer les trois degrés que sont la «totalité in-achevée », la «particularité pleinementachevée » et la «totalité pleinement-achevée ${ }^{18}$. Pour obtenir un système des valeurs, il faut en réalité adjoindre à cette hiérarchie et croiser avec elle un ordre typologique des biens culturels eux-mêmes, qui sont l'étoffe même du monde historique, ordre fondé sur des alternatives hétérologiques superposables comme chose / personne, contemplation / activité ou social / $\operatorname{asocial}^{19}$. De ce croisement résulte un système de six domaines axiologiques fondamentaux répartis symétriquement de part et d'autre des alternatives mentionnées (science, art et panthéisme mystique du côté contemplatif, vie socio-éthique, amour personnel et religion théiste du côté actif). Les structures hétérologiques de la réalité historique sont ainsi des composantes nécessaires du système des valeurs. C'est la raison pour laquelle Rickert peut affirmer que ce système repose sur « des facteurs qui, tout en surplombant toute histoire, n'entrent pas pour autant en conflit avec elle $»^{20}$.

Cette absence de conflit se traduit dès lors positivement par une caractéristique fondamentale du système, que Rickert se plait à souligner : son ouverture. De fait, le caractère formel de cette construction qui échappe pourtant à tout formalisme (puisqu'elle intègre la typologie des biens culturels en tant qu'effectivités historiques) garantit à la fois sa validité définitive et sa capacité à intégrer les produits de l'évolution à jamais ouverte de l'histoire et de la culture, qu'il s'agisse de biens à venir ${ }^{21}$ ou de valeurs inédites ${ }^{22}$. Le propre du système

\footnotetext{
${ }^{17}$ H. RICKERT, « Le système des valeurs », trad. cit., p. 139.

${ }^{18}$ Cf. Ibid., p. 141-142.

${ }^{19}$ Cf. Ibid., p. $144 s q$.

${ }^{20}$ Ibid., p. 136.

${ }^{21}$ Cf. Ibid., p. 139 : « il reste pourtant de la place pour la multitude non-close des biens culturels historiques ».

${ }^{22}$ Cf. Ibid., p. 147 : «il reste en même temps de la place pour les valeurs de la culture qu'une évolution historique ultérieure peut éventuellement porter à notre conscience ». Pour être tout à fait précis, il faut noter que c'est bien en tant que système des valeurs que le système est ouvert; c'est la raison pour laquelle Rickert n'entreprend pas de fixer le nombre des valeurs mais seulement des domaines axiologiques fondamentaux, dont le nombre est, pour le coup, absolument intangible, et relativement auxquels le système est parfaitement clos.
} 
des valeurs est donc d'être un système ouvert en dépit de sa clôture formelle, d'être un système dont l'achèvement formel n'a de sens et de légitimité que de fixer pour ainsi dire son ouverture à tout ce que l'évolution historique est susceptible de produire en terme de configurations culturelles. Le point important est ici que ce caractère ouvert du système des valeurs, loin d'être le corollaire d'une quelconque indétermination dans les principes de son édification, est la contrepartie philosophique positive de la non-fermeture (Unabgeschlossenheit) de l'histoire elle-même ${ }^{23}$, c'est-à-dire de cette détermination fondamentale qui lui revient dès lors qu'on la pense à partir de l'avenir et non pas de façon seulement cumulative à partir du passé. En ce sens, le système des valeurs est donc bien un système de l'histoire ou de la vie historique, car il est le seul système qui non seulement convienne à l'histoire dans sa dimension asystématique, mais qui procède paradoxalement de cette asystématicité même.

Si le système des valeurs signifie en lui-même une critique de l'historicisme, c'est donc parce qu'il est doublement articulé à l'effectivité du monde historique concret, de telle sorte qu'il fait voler en éclats l'opposition traditionnelle entre l'historique et le systématique : non seulement il procède de l'histoire puisque les valeurs ne peuvent être découvertes qu'à même les biens historico-culturels, mais il ouvre sur elle et sur ce qu'il y a de plus essentiel en elle, à savoir son ouverture même. Le système des valeurs fournit ainsi l'illustration, si ce n'est la preuve, que l'abandon de toute systématicité n'est pas la réponse philosophique adéquate à une prise en considération du champ de l'effectivité historique : une systématicité axiologique peut encore en procéder. Mais on voit immédiatement surgir ici l'insuffisance de cette critique : de ce qu'une philosophie systématique des valeurs peut et même doit procéder d'une thématisation du monde de l'effectivité historique, il ne résulte pas que cette exigence provienne de l'histoire elle-même. Qu'il n'y ait pas de philosophie des valeurs ineffectives sans histoire est une chose ; mais que l'histoire ne soit pas concevable sans une philosophie des valeurs ineffectives en est assurément une autre. En ce sens, que le système des valeurs soit enraciné dans l'histoire n'exclut pas qu'il soit en réalité greffé sur elle comme un élément étranger à son essence. Par suite, si le système des valeurs présente bien des armes critiques contre l'historicisme, elles demeurent absolument inoffensives tant qu'il n'est pas montré que l'histoire elle-même exige une philosophie des valeurs transcendantes, ou qu'une telle

L'idée de Rickert est que les valeurs que l'humanité sera amenée à reconnaître dans la suite de son évolution historique relèveront nécessairement, quelles qu'elles soient, de l'un ou de l'autre des six domaines mentionnés. ${ }^{23}$ Cf. Ibid., p. 136, 139. 
philosophie répond effectivement à une détermination essentielle de l'histoire. C'est ce qu'il nous faut donc examiner à présent dans un deuxième temps de notre démarche.

Que l'histoire exige le développement d'une philosophie des valeurs, c'est là un point qui est acquis très tôt par Rickert sur le seul versant épistémologique de son œuvre, puisque c'est à vrai dire le but explicite de l'argumentation des Grenzen depuis leur première édition ainsi que du célèbre ouvrage intitulé Science de la culture et science de la nature, lui aussi constamment réédité par Rickert entre 1899 et $1926^{24}$. Comme il s'agit là sans aucun doute de l'aspect le plus connu et le plus discuté de l'œuvre de Rickert (à défaut peut-être d'être le plus intéressant en lui-même), nous nous contenterons ici d'en rappeler brièvement le contenu. Dans cette perspective exclusivement épistémologique, la "philosophie de l'histoire » est considérée « comme la science du savoir historique, ou comme une partie de la logique dans l'acception la plus large du terme $»^{25}$, c'est-à-dire encore comme une méthodologie de la connaissance historico-culturelle. Son objectif est d'exhiber le fondement de l'opposition des sciences historiques aux sciences de la nature et, comme on le sait, le propre de la démarche de Rickert (par opposition à celle de Dilthey ou de Husserl) est de ressaisir l'essence logique d'une science à partir du but logico-théorique qu'elle se propose et du type de formation de concepts (Begriffsbildung) qui en découle nécessairement à titre de moyen. La thèse de Rickert est connue: prolongeant l'intérêt préscientifique pour l'individuel qui compose comme tel notre monde environnant, les sciences historiques visent une connaissance conceptuelle de l'individualité et mettent ainsi en œuvre une méthode individualisante qui transforme la réalité préscientifique, à la fois continue et hétérogène, en un discret hétérogène $\mathrm{e}^{26}$. Or le point décisif est que ce qui fait de tel ou tel aspect de la réalité préscientifique un individu au sens où les sciences historiques l'entendent, n'est pas l'une ou l'autre de ses caractéristiques factuelles mais uniquement la valeur qui lui est associée. C'est seulement la "relation à la valeur » (Wertbeziehung) qui fait des individualités historiques (qui ne sont pas nécessairement des faits singuliers isolés mais aussi et surtout des touts

\footnotetext{
${ }^{24}$ H. RICKERT, Kulturwissenschaft und Naturwissenschaft, Freiburg, Leipzig, Tübingen, J. C. B. Mohr (Paul Siebeck), ${ }^{1} 1899,{ }^{2} 1910,{ }^{3} 1915,{ }^{4-5} 1921,{ }^{6-7} 1926$; trad. fr. par A.-H. Nicolas, Science de la culture et science de la nature, op. cit.

${ }^{25}$ H. RICKERT, Les problèmes de la philosophie de l'histoire, op. cit., p. 55.

${ }^{26}$ Cf. H. RICKERT, Science de la culture et science de la nature, op. cit., p. 60-61.
} 
historiques $^{27}$ ) ce qu'elles sont, et qui est en même temps pour l'historien le principe méthodologique fondamental de sélection de son objet dans la matière historique, inépuisable comme telle. Cette axiologie historique est donc seulement théorique et doit bien être distinguée de toute prise de position évaluative pratique de la part de l'historien : elle n'a d'autre effet, comme dit Rickert dans les Grenzen, que « de séparer le matériel, qui est pris en considération historiquement, du réel qui reste au contraire sans importance su point de vue théorique $»^{28}$. Simultanément, ce rapport à la valeur permet de définir le monde historique comme l'ensemble des réalités signifiantes susceptibles d'être comprises, par opposition à la nature comme ensemble légal homogène des réalités dépourvues de signification.

Si l'histoire appelle donc une thématisation philosophique des valeurs en tant qu'idéalités distinctes non seulement des réalités auxquelles elles sont susceptibles d'être associées mais aussi des sujets qui les y associent dans leurs actes d'évaluation, c'est d'abord dans la mesure où la méthode historique implique inévitablement une référence constante à de telles valeurs. Mais on voit rapidement que ce résultat (auquel on réduit trop fréquemment la philosophie rickertienne) est tout à fait insuffisant au regard des questions qui ont émergé à l'issue de notre présentation de l'idée d'un système des valeurs, et ce pour deux raisons principales qui sont intimement liées. D'une part, il n'est toujours question que de la science historique et rien n'a encore été dit de l'histoire comme ce dont cette science est l'étude, ni par conséquent de l'articulation de cette histoire avec une philosophie des valeurs. D'autre part, il faut noter qu'une telle délimitation logico-méthodologique de la connaissance historique ne peut fonder pour cette dernière qu'une objectivité limitée, dans la mesure où elle ne permet pas de rendre raison des présupposés que l'historien est contraint d'accomplir :

l'historien, comme tel, n'a pas à s'interroger sur la validité des valeurs qui dirigent sa représentation. Il ne rapportera pas pour autant ses objets à n'importe quelles valeurs. Il présuppose au contraire que le public auquel il destine ses représentations reconnaîtra comme valeur, ou en tout cas comprendra

\footnotetext{
${ }^{27}$ C'est un point qu'il convient de souligner, bien que nous ne puissions ici le développer plus amplement. La conception rickertienne de l'histoire comme science individualisante n'implique aucune conception atomiste de la réalité historique : les individualités étudiées pas l'historien ne sont pas seulement des singularités séparées les unes des autres, mais aussi et surtout des ensembles ou des contextes (Zusammenhang) historiques, qui sont autant de milieux évolutifs. Pour l'historien, l'individualité qui est l'objet de son l'investigation est ainsi au premier chef celle d'une «série évolutive ». Par conséquent, la méthode individualisante de l'histoire n'exclut absolument pas qu'il y soit question de généralité ; il s'agit au contraire pour le théoricien de la science d'apercevoir qu'il y a différents sens de la généralité, et parmi eux un sens qui convient à la réalité historique dans son caractère pourtant essentiellement individuel, et qui se distingue logiquement de la généralité naturelle. De même, si l'historien peut quant à lui parfaitement faire usage de concepts généraux, cet usage ne se laisse pas identifier avec celui qu'en font les chercheurs en science de la nature. Sur tous ces points que nous ne pouvons qu'évoquer, on se reportera par exemple à l'exposé fourni par Rickert dans Les problèmes de la philosophie de l'histoire, op. cit., p. 86-94.

${ }^{28}$ H. RICKERT, Die Grenzen..., ${ }^{3} 1921$, op. cit., p. 251, cité par M. FERRARI, op. cit., p. 129 ; cf. également Science de la culture et science de la nature, op. cit., p. 125 et Les problèmes de la philosophie de l'histoire, op. cit., p. 99-100.
} 
comme valeur, ainsi que lui-même, sinon tels ou tels biens particuliers, du moins les valeurs générales de la religion, de l'État, du droit, des mœurs, de l'art, de la science, par rapport auxquelles ce qu'il représente historiquement est essentiel ${ }^{29}$.

La pratique théorique de l'historien est ainsi cernée par deux présuppositions : en amont, celle de la validité en soi des valeurs qui lui servent de principe de sélection pour retenir ce qui est essentiel au regard de son projet théorique; en aval, celle de leur validité pour une communauté intersubjective, validité qui seule rendra en retour son travail d'historien compréhensible et communicable. Avec cette double présupposition se dessine le problème de l'universalité des valeurs qui guident la science historique, et par conséquent le problème du type d'objectivité dont elle est susceptible. Et en vertu de la deuxième présupposition, il semble que cette objectivité ne puisse être que relative, étant donné que les valeurs ne peuvent être présupposées comme valides que pour une communauté culturelle empiriquement déterminée, qui peut être au mieux, comme l'énonce Rickert dans une formule éloquente dans son imprécision même, «un ensemble d'hommes relativement important " ${ }^{30}$. Même si tout arbitraire se trouve ici exclu par le fait que les valeurs en question sont simplement formelles et indépendantes de toute particularisation matérielle dans le temps et l'espace, il reste elles ne peuvent fonder qu'une objectivité empirique pour le discours de l'historien.

Or il est tout à fait décisif de remarquer l'historien n'est contraint d'effectuer cette seconde présupposition, en vertu de laquelle il ne saurait dépasser une objectivité seulement empirique, que parce qu'il n'a pas les moyens théoriques d'éviter la première présupposition, qui concerne la validité en soi des valeurs. Inversement, satisfaire à l'exigence non historique d'une étude systématique de cette validité supra-historique des valeurs est le seul moyen d'assurer à l'histoire comme science une objectivité plus qu'empirique ${ }^{31}$. C'est ainsi que l'exigence d'un système des valeurs se profile à l'horizon du versant épistémologique de la philosophie rickertienne, afin de garantir pour la science historique une objectivité qui ne soit pas seulement de fait, ce qu'une simple logique de la connaissance historique n'est pas en mesure de fournir. Que cette exigence soit proprement philosophique et non historique, on en conviendra aisément; la question, de nouveau, est celle de savoir en quoi il s'agit là d'un problème de philosophie de l'histoire. Mais nous avons maintenant les moyens de répondre à cette question, et paradoxalement à partir du résultat principal des recherches logicométhodologiques elles-mêmes.

\footnotetext{
${ }^{29}$ H. RICKERT, Science de la culture et science de la nature, op. cit., p. 135.

${ }^{30}$ Ibid., p. 181.

${ }^{31}$ Cf. Ibid., p. 183.
} 
Ce résultat prend la forme de la thèse selon laquelle « l'axiologie est la condition sine qua non de la conception individualisante [c'est-à-dire historique] en soi $»^{32}$. Si tout travail historique consiste à dégager et à décrire, moyennant l'axiologie théorique comme méthode individualisante, des ensembles historiques cohérents sous la forme de «séries évolutives uniques et individuelles $»^{33}$, un système des valeurs formelles et absolument universelles présupposées par toute axiologie historique possible doit donc être considéré en lui-même comme le principe d'une thématisation de l'histoire elle-même en tant qu'objet total et individuel de toute science historique, ou, pour utiliser la terminologie de Rickert, en tant qu'universum historique. Ce que Rickert veut dire par là, c'est avant tout que l'histoire comme objet d'une philosophie de l'histoire n'est rien d'autre que l'histoire telle qu'elle s'élabore dans les sciences historiques, et qu'il n'y a pas à proprement parler d'histoire en soi :

Nous devons entendre par «histoire» ce déroulement unique de la vie culturelle signifiante et axiologique tel qu'il est décrit de façon individualisante par l'ensemble de toutes les disciplines historiques $[\ldots]^{34}$.

Cette histoire [...] est, pour la philosophie, identique à l'histoire en soi, et ce sont ses principes qui intéressent la philosophie de l'histoire ${ }^{35}$.

S'il n'y a donc pas d'histoire en dehors des sciences historiques, il reste que ces dernières, en raison de leur diversité et de l'éparpillement de la matière historique en elles, n'épuisent pas complètement leur objet dans la mesure où elles ne peuvent pas rendre compte par ellesmêmes de «la totalité de l'histoire conçue comme une unité » ${ }^{36}$. C'est donc à la philosophie qu'il revient de thématiser cette totalité idéale en en fournissant le principe de cohérence interne, présupposé par toute histoire empirique. À cette double exigence idéale de totalité et de cohérence vient répondre le système - idéal, lui aussi - des valeurs, dont l'édification constitue la tâche infinie d'une philosophie de l'histoire qui demeure ainsi parfaitement conforme à son acception traditionnelle de doctrine $d u$ sens de l'histoire, puisque ce sont les valeurs qui sont au principe de tout sens compréhensible en général. Conçue non plus seulement comme historia rerum gestarum mais comme totalité signifiante en devenir, l'histoire appelle donc bien une philosophie systématique des valeurs.

Dans cette mesure, l'exigence d'un système des valeurs est à la fois nécessaire et suffisante pour délimiter le domaine théorique précis de la philosophie de l'histoire par

\footnotetext{
${ }^{32}$ H. RICKERT, Les problèmes de la philosophie de l'histoire, op. cit., p. 139.

${ }^{33}$ Ibid., p. 89.

${ }^{34}$ Ibid., p. 123

${ }^{35}$ Ibid., p. 122-123.

${ }^{36}$ Ibid., p. 123.
} 
opposition à la simple logique de la connaissance historique, laquelle semblait pouvoir revendiquer aussi pour elle-même ce titre. Que manque-t-il donc, en toute rigueur, à la démarche logico-méthodologique pour être une philosophie de l'histoire au sens plein du terme, et quel manque vient combler l'édification d'un système des valeurs? La réponse de Rickert est claire : le système des valeurs permet à la philosophie de l'histoire de se présenter non pas seulement comme une analytique mais aussi comme une critique, et ce en un double sens. Elle est tout d'abord critique au sens kantien et fondationaliste du terme dans la mesure où elle garantit pour les sciences historiques la possibilité d' " une “objectivité" en un sens qui n'est plus simplement empirique $»^{37}$. Mais elle est aussi critique au sens où la systématique des valeurs supra-historiques lui donne la capacité d'évaluer toutes les « tentatives qui dépassent l'histoire empirique dans la mesure où elles veulent explicitement poser les principes de la vie historique $»^{38}$. Le système des valeurs fait ainsi de la philosophie de l'histoire un tribunal des discours axiologiques statuant sur l'essence de l'histoire prise comme totalité, dont Rickert privilégie souvent trois exemples : le matérialisme historique de Marx, le positivisme de Comte ou le naturalisme biologiste de Spengler. Guidée par le système des valeurs, cette philosophie critique de l'histoire peut dès lors prendre deux formes : soit la mise en évidence des présupposés axiologiques qui guident secrètement un discours prétendant exhiber des lois anaxiologiques de l'histoire sur le modèle des sciences de la nature (c'est le cas de Comte et de Spengler) ${ }^{39}$, soit la mise en évidence de l'absolutisation unilatérale d'une valeur particulière au principe d'un discours censé valoir pour toutes les dimensions de la vie historique (c'est le cas de Marx) ${ }^{40}$.

\footnotetext{
${ }^{37}$ H. RiCKERT, Science de la culture et science de la nature, op. cit., p. 187 (trad. légèrement modifiée).

${ }^{38}$ H. RICKERT, Les problèmes de la philosophie de l'histoire, op. cit., p. 142.

${ }^{39}$ La critique de Comte s'effectue dans le cadre de la démonstration que des lois, au sens strict du terme, ne sauraient fournir les principes de l'histoire conçue comme unité (cf. Ibid., p. 126-132, 136-137). La critique de Spengler est accomplie pour sa part d'une double manière : soit directement à partir de l' «opposition principale » entre méthode généralisante et méthode individualisante (c'est alors le naturalisme méthodologique de Spengler qui est réfuté - cf. H. RICKERT, Science de la culture et science de la nature, op. cit., p. 32, note) ; soit indirectement, dans le cadre de la critique de la «philosophie de la vie », moyennant la réfutation de la thèse selon laquelle la vie biologique constitue par elle-même une valeur, au profit de la thèse selon laquelle les valeurs de vie ne sont pas des valeurs en propre (Eigenwerte) mais des valeurs conditionnelles (Bedingungswerte), c'est-à-dire des valeurs qui ne sont que les présuppositions de la réalisation de valeurs authentiques (c'est alors le biologisme de Spengler qui est pris en considération - cf. sur ce point l'article "Valeurs de vie et valeurs de culture », trad. fr. par nos soins in H. RICKERT, Le système des valeurs et autres articles, op. cit., en part. p. 115-118 et 131-132). Notons que ces deux directions de la critique de Spengler sont reprises dans le célèbre ouvrage polémique de Rickert, Die Philosophie des Lebens. Darstellung und Kritik der philosophischen Modeströmungen unserer Zeit, Tübingen, J. C. B. Mohr (Paul Siebeck), ${ }^{1} 1920,{ }^{2} 1922$, p. 32 pour la première, p. 117-130 et 135-136 pour la seconde.

${ }^{40}$ Cette critique permet à Rickert d'établir a contrario la nécessité d'un système des valeurs universelles au principe de la philosophie de l'histoire (cf. H. RICKERT, Les problèmes de la philosophie de l'histoire, op. cit., p. $142 s q$.$) .$
} 
Cette présentation, quoique schématique, permet dès lors de préciser la nature de la critique rickertienne de l'historicisme telle que nous l'avons vu émerger dans la seule définition théorique du système des valeurs. Dans la mesure où le système des valeurs exhibe les « conditions axiologiques » non seulement de la connaissance historique mais de l'histoire elle-même comme totalité singulière, on peut dire que la démarche de Rickert consiste à combattre l'historicisme par le moyen de l'histoire elle-même, de sorte que l'anti-historicisme prend ici paradoxalement la forme d'un «combat pour une appréhension historique de la réalité et pour le droit de l'histoire ${ }^{41}$. Dans ce contexte, la réfutation de l'historicisme est une réfutation par les effets : c'est le relativisme induit par l'historicisme qui est déraciné s'il est montré que l'historique lui-même exige des valeurs anhistoriques à titre de condition d'intelligibilité. Simultanément, la mise au jour de cette exigence permet de ressaisir l'unité de la philosophie rickertienne des valeurs : non seulement parce que le système des valeurs s'y atteste comme le lieu de jonction de la théorie de la connaissance et de la théorie de la science, mais plus radicalement parce que philosophie des valeurs et philosophie de l'histoire deviennent coextensives. En effet, et contrairement à ce qu'avance Raymond Aron dans sa présentation de la philosophie rickertienne de l'histoire, celle-ci n'est pas incluse dans la philosophie des valeurs comme une de ses parties ${ }^{42}$, mais les deux se superposent dans la mesure où leurs fondements respectifs coïncident véritablement :

La réflexion sur les valeurs supra-historiques n'est plus du ressort de la philosophie de l'histoire conçue comme une spécialité [...]: on ne peut l'entreprendre que dans le contexte de l'élaboration d'un système de philosophie générale. [...] Les fondements de la philosophie de l'histoire coïncident donc avec les fondements de la philosophie en tant que science axiologique ${ }^{43}$.

Cette coïncidence permet dès lors de rendre compte d'un point qui n'est pas relevé par Aron et qui mérite pourtant un commentaire, à savoir l'absence frappante de toute mention de l'histoire de la part de Rickert au moment de la détermination des domaines axiologiques fondamentaux qui structurent le système des valeurs. Il faut comprendre en réalité que l'histoire ne peut en aucun cas être considérée comme un domaine axiologique, mais ce non pas parce qu'elle serait le lieu de dissolution de toute validité universelle, mais bien plutôt parce que loin d'être un domaine axiologique parmi d'autres, elle est ce dont le sens vient s'articuler dans le système des valeurs à travers ces domaines axiologiques particuliers. Comprise comme universum historique, l'histoire est en quelque sorte le système des valeurs

\footnotetext{
${ }^{41}$ H. RICKERT, Science de la culture et science de la nature, op. cit., p. 190.

${ }^{42}$ R. ARON, La philosophie critique de l'histoire. Essai sur une théorie allemande de l'histoire, Paris, Vrin, 1969, rééd. Seuil, 1970, p. 135.

${ }^{43}$ H. RICKERT, Les problèmes de la philosophie de l'histoire, op. cit., p. 147.
} 
lui-même comme structuration dynamique et ouverte de la validité en soi, si bien que la discipline qui se propose de mettre au jour cette systématicité est eo ipso une doctrine des principes axiologiques de la totalité historique.

Il reste que pour Rickert, une telle doctrine n'épuise pas le sens d'une philosophie de l'histoire. Elle ne constitue en réalité pour lui qu'une étape préparatoire de la philosophie de l'histoire au sens le plus haut, et ne doit être comprise par suite que comme une «prolégoménologie » ${ }^{44}$, c'est-à-dire comme un simple préambule méthodologique appartenant certes lui-même à la philosophie de l'histoire. Il nous faut donc, dans un dernier temps, suivre Rickert dans sa détermination du sens éminent de la philosophie de l'histoire pour voir quel rôle y joue le système des valeurs et répondre plus profondément aux questions directrices que nous avons soulevées en introduction.

Prise en son sens le plus ambitieux, la philosophie de l'histoire se veut elle-même une « histoire générale » ou une « histoire mondiale » ${ }^{45}$ - bref, une « histoire universelle », c'està-dire non pas « une coupe transversale de l'histoire dans son ensemble, mais une coupe longitudinale $»^{46}$. Or pour Rickert, un tel projet, s'il a un sens légitime, ne saurait se concevoir comme celui d'une synthèse sommative de toutes les histoires particulières ni encore moins comme une construction d'hypothèses spéculatives destinées à compléter le travail des historiens. Ces dénégations, qui peuvent paraître autant d'évidences, sont en réalité les effets d'une thèse rickertienne à la fois simple et profonde, et qui n'est elle-même que le prolongement du refus de toute histoire en soi, que nous avons rencontré plus haut : la thèse selon laquelle ce sont les historiens qui écrivent au premier chef l'histoire universelle ${ }^{47}$, de sorte que quand celle-ci devient une tâche philosophique, elle ne peut pas être radicalement étrangère à l'histoire écrite par les historiens, qui doit bien plutôt en être la matière première ${ }^{48}$. En quoi consiste dès lors la philosophie de l'histoire en ce sens ? La réponse la plus claire est

\footnotetext{
${ }^{44}$ Ibid., passim.

${ }^{45}$ Ibid., p. 54.

${ }^{46}$ Ibid., p. 149.

${ }^{47}$ Cf. Ibid., p. 149-150 : « les historiens eux-mêmes écrivent l'histoire universelle formulant nécessairement tout ce qui ressortit à la problématique d'une "histoire générale" ».

${ }^{48}$ Il s'agit avec elle «d'un traitement philosophique de ce même objet que les sciences de l'histoire décrivent historiquement » (Ibid., p. 154).
} 
fournie par Rickert dans un texte qui l'articule avec la conception prolégoménologique de la philosophie de l'histoire, telle que nous l'avons évoquée plus haut :

On devra d'abord, indépendamment de la diversité du matériau historique, réfléchir à ce qui est nécessairement valable, à ce qui est prémisse formelle de tout jugement de valeur qui prétend à une validité plus qu'individuelle. C'est seulement quand on aura trouvé des valeurs formelles valables pour toutes les époques qu'on pourra les mettre en rapport avec la quantité de valeurs culturelles au cours de l'histoire et qu'on peut constater empiriquement. On pourra alors tenter un classement systématique assorti d'une prise de position critique ${ }^{49}$.

Quoique programmatique, ce texte permet de saisir une divergence et un point commun entre les deux formes de la philosophie de l'histoire. La divergence concerne le rapport à l'effectivité historique : alors que la prolégoménologie cherche, par abstraction à partir du contenu de l'histoire effective, à dégager les principes anhistoriques de l'universum historique comme totalité individuelle, l'histoire universelle tente de rattacher ces valeurs universelles anhistoriques aux valeurs culturelles effectivement réalisées, telles que l'historien apprend à les connaître. Le point commun vient quant à lui du fait que dans les deux cas, on se trouve en présence d'une discipline tout à la fois analytique et critique.

Pour préciser ce dernier aspect, il faut noter que de même que le système des valeurs permettait d'isoler la philosophie de l'histoire comme prolégoménologie par rapport à la simple logique de la connaissance historique, c'est encore lui qui permet de saisir à présent comment ce premier sens de la philosophie de l'histoire s'articule au sens éminent dont il est question à présent. La prolégoménologie prépare un système des valeurs en vue de son utilisation par la philosophie de l'histoire au sens éminent, laquelle produit donc une histoire universelle par évaluation de l'histoire effective au moyen de ce système. Deux conséquences doivent dès lors être indiquées. D'une part, l'histoire universelle chez Rickert n'est pas la récriture philosophique de l'histoire, mais «la description philosophique systématique de l'universum qui ne se limite pas aux valeurs formelles mais les met expressément en rapport avec le contenu de l'être historique lui-même ${ }^{50}$. Mais, d'autre part, cette analyse descriptive ne peut être simultanément qu'une évaluation critique, au sens où l'utilisation herméneutique du système des valeurs permet non seulement de dégager des époques et des périodes qui ne coïncident pas nécessairement avec celles que les historiens exhibent, mais surtout de voir en ces époques les étapes d'un progrès à l'œuvre dans l'histoire. Cela précisé, une question se pose encore : de quoi ces époques et ce progrès sont-ils les époques et le progrès ? Ou encore, plus généralement : de quoi l'histoire universelle philosophique est-elle ainsi l'histoire ?

\footnotetext{
${ }^{49}$ Ibid., p. 146.

${ }^{50}$ Ibid., p. 172.
} 
À quoi il faut répondre qu'en tant qu'éclairage interprétatif de l'effectivité historique sous la conduite du système des valeurs, l'histoire universelle rickertienne est l'esquisse de l'histoire, à jamais inachevée, de la réalisation progressive des valeurs transcendantes dans le monde culturel humain, c'est-à-dire à même les événements historiques que les historiens nous font connaître. Si, en tant que prolégoménologie, la philosophie de l'histoire livre dans le système des valeurs le sens abstrait de l'universum historique, elle cherche, en tant qu'histoire universelle, à présenter le sens concret de cet universum sous la forme nécessaire (et moins paradoxale qu'il ne paraît) d'une "description axiologique » de l'effectivité historique ${ }^{51}$ : elle se sert pour cela du système des valeurs comme d'un outil de lecture de l'histoire effective pour y identifier les époques de la réalisation des valeurs transcendantes. C'est ainsi, par exemple, que la naissance de la philosophie en Grèce antique est une époque fondamentale de l'histoire universelle dans la mesure où c'est le moment où cette valeur théorique qu'est la vérité se trouve réalisée dans la conception de la science comme theoria ${ }^{52}$. De même, ainsi que l'ont montré Kant et les philosophes idéalistes allemands, la Révolution française a une signification historique universelle du point de vue de la réalisation effective de la valeur éthico-politique qu'est la liberté ${ }^{53}$.

Au-delà de ces exemples, on voit que le dépassement de l'historicisme est ici beaucoup plus radical que dans la seule prolégoménologie, et qu'il repose précisément sur le caractère normatif des descriptions qui sont rendues possibles dans ce contexte. Toutes les époques ne se valent pas ; certaines sont décisives, d'autres sont négligeables eu égard à la réalisation de valeurs dont l'universalité anhistorique prévient tout arbitraire spéculatif de la part du philosophe de l'histoire. Dans ces conditions, on peut dire de la critique rickertienne de l'historicisme qu'elle procède à une inversion de la célèbre formule schillerienne : loin d'être elle-même le tribunal, l'histoire est citée à comparaître devant le tribunal de la philosophie qui met en œuvre le système des valeurs dans une interprétation axiologique de la réalité historique. Comme l'écrit Rickert :

Elle [sc. la philosophie de l'histoire] doit se démarquer consciemment de l'observation historique pure, et non seulement médiatiser le passé en faveur du présent et de l'avenir, mais aussi le « juger », c'est-à-

\footnotetext{
${ }^{51}$ Cette expression n'est pas utilisée, à notre connaissance, par Rickert, mais les développements qu'il consacre à la tâche programmatique de ce qu'il nomme "histoire universelle philosophique » (cf. par exemple Ibid., p. 153 sq.) nous semblent la justifier amplement.

52 Nous renvoyons sur ce point à l'article de 1923-1924 intitulé «La vie de la science et la philosophie grecque », trad. fr. par nos soins in H. RICKERT, Le système des valeurs et autres articles, op. cit., en part. p. 186191.

${ }^{53}$ H. RICKERT, Les problèmes de la philosophie de l'histoire, op. cit., p. 164-165.
} 
dire l'évaluer avec des critères normatifs. Il n'existe pas d'autre voie pour dépasser le relativisme et le nihilisme de l'historicisme ${ }^{54}$.

Avec cette suspension de la neutralité axiologique du discours sur l'histoire, la réfutation de l'historicisme atteint ici sa forme la plus achevée et la plus subtile, dans la mesure où elle ne consiste pas à supprimer tout simplement l'opposition entre l'historique et le systématique, contrairement à ce que pouvait faire penser la définition théorique et abstraite du système des valeurs. Il ne s'agit pas non plus d'inverser schématiquement les pôles en historicisant la philosophie et en systématisant l'histoire. Cette opposition se trouve bien plutôt ravalée à un simple constat factuel, privé en soi de toute signification philosophique; elle est donc parfaitement corroborée et même justifiée par Rickert. La philosophie ne peut faire autrement, si elle veut rester philosophie, que de traiter l'histoire a-systématique de façon a-historique, c'est-à-dire sur le fondement de valeurs universelles dégagées quant à elles à partir de l'histoire. Au fond, Rickert cherche à montrer que l'interprétation axiologique (c'est-à-dire systématique et critique) de l'histoire est la réponse adéquate que la philosophie apporte à la question que lui adresse l'histoire, et ce à partir des matériaux qu'elle lui présente. C'est sa systématicité a-historique qui permet à la philosophie de satisfaire à l'universalité qui s'annonce dans l'historicité a-systématique du matériau historique. Quand donc Rickert dit de sa philosophie qu'elle est « orientée sur l'histoire ${ }^{55}$, il faut donc comprendre que seule une philosophie systématique des valeurs peut l'être et qu'elle l'est même doublement : orientée sur elle pour s'élever à partir d'elle à la systématicité de la sphère axiologique, et orientée sur elle dans le retour interprétatif dont procède l'histoire universelle en tant qu'interprétation axiologique de l'histoire effective.

Ces remarques nous permettent à présent de comprendre le lien profond entre le thème de l'histoire et la question de l'unité de la philosophie de Rickert en tant que criticisme axiologique, au-delà même de la distinction entre théorie de la connaissance et théorie de la science. Il nous semble en effet qu'une mésinterprétation plus grave encore que l'autonomisation complète de ces deux versants menace tout discours sur la philosophie de Rickert en général - celle en vertu de laquelle cette pensée s'épuiserait dans la mise au jour d'une sphère systématique de la validité pure. Il ne s'agit évidemment pas de nier que la philosophie de Rickert soit bel et bien une «philosophie des valeurs »; elle l'est assurément, pourvu toutefois qu'on n'entende pas seulement par là la mise au jour d'une sphère de valeurs ineffectives et transcendantes, mais aussi et surtout, comme Rickert ne cesse de l'affirmer

\footnotetext{
${ }^{54}$ Ibid., p. 157.

${ }^{55}$ Cf. supra, note 10.
} 
dans tous ses livres et ses articles, la doctrine de l'articulation de ces valeurs ineffectives et de l'effectivité psychophysique du monde réel. L'objet dernier des recherches de Rickert n'est pas tant la validité comme telle que son articulation avec l'effectivité au sein d'une sphère intermédiaire qu'il nomme le «troisième règne ${ }^{56}$. Autrement dit, philosophie des valeurs et axiologie ne sont pas coextensives, et le problème qui est au foyer de la philosophie de Rickert en tant que philosophie des valeurs est celui de la juste articulation de l'axiologique et de l'ontologique. Or nos analyses montrent que de ce point de vue aussi, la philosophie des valeurs est doublement orientée sur l'histoire. En premier lieu, dans la mesure où elle est la totalité culturelle des biens, des effectivités pourvues de valeurs, l'histoire atteste de facto qu'une unité des deux règnes fondamentaux de l'être et de la validité est nécessairement possible. Mais comme le dit Rickert, le « principe du rattachement $~^{57}$ des deux règnes n'est pas encore fourni par là. Beaucoup plus large que le simple «fait des sciences » des néokantiens de l'école de Marbourg, cette unité joue donc le rôle, chez Rickert, de factum total dont il s'agit d'exhiber les conditions transcendantales de possibilité. En second lieu, si l'on se souvient qu'en 1911, Rickert nomme le troisième règne celui du «sens » et « interprétation » 1 'acte par lequel on entre dans un tel règne du sens ${ }^{58}$, il devient évident que la philosophie de l'histoire en son acception la plus élevée fournit une telle élucidation du troisième règne dans la mesure où elle n'est rien d'autre que l'interprétation du sens de la réalité historique sur le fondement de valeurs universellement valides.

Ainsi notre parcours confirme-t-il que la signification et la portée philosophiques de l'idée rickertienne d'un système des valeurs ne sauraient être appréciées indépendamment de la définition renouvelée de la philosophie de l'histoire dans le contexte théorique d'un idéalisme axiologique. De ce point de vue, et quel que soit l'intérêt des analyses qui y sont menées, il faut reconnaître que l'article de 1913 que nous avons commenté en première partie souffre d'une double insuffisance : sans lien apparent avec la problématique de la philosophie de l'histoire, il n'est pas sans donner l'impression d'une construction théorique purement

\footnotetext{
${ }^{56}$ Cf. H. RiCKERT, « Le concept de la philosophie », trad. cit., p. 72 sq. Pour des précisions sur cette notion dans le contexte de la discussion critique que Rickert entretient à son propos avec son disciple Emil Lask, nous nous permettons de renvoyer aux indications que nous donnons dans en présentation, Ibid., p. 24-26.

${ }^{57}$ Ibid., p. 77.

${ }^{58}$ Cf. Ibid., p. 80.
} 
formelle et gratuite ${ }^{59}$, qui trahirait de façon plus ou moins heureuse l'attachement indéfectible de Rickert à la tradition des grands systèmes idéalistes post-kantiens ; et si une lecture plus attentive découvre qu'il n'est pas sans faire place au thème général de l'histoire, il reste que sa contribution est purement négative puisqu'elle se limite à une critique, largement implicite, de l'historicisme. La signification fondamentale du système des valeurs ne sera exposée que dans les textes des années 20, où il est expressément mis en relation avec la question de la philosophie de l'histoire. Il n'est d'ailleurs pas exclu que ce soit précisément l'approfondissement de l'idée d'un système ouvert des valeurs qui ait conduit Rickert à déterminer de façon toujours plus précise le sens que prend la philosophie de l'histoire dans le dans le cadre général de sa philosophie des valeurs. Et de fait, nos analyses ont montré que le système des valeurs contribue à cette détermination d'une double manière.

D'un côté, il est apparu que le système des valeurs permettait de construire un concept différencié de la philosophie de l'histoire, renvoyant à des niveaux distincts de recherches théoriques, qui correspondent à différents types de mises en œuvre de ce système. Conçu comme la présentation ordonnée des conditions axiologiques sans lesquelles l'histoire n'est pas pensable comme totalité singulière (comme universum historique), le système des valeurs est le moment essentiel d'une philosophie de l'histoire comprise comme doctrine des principes de la vie historique, vouée à la thématisation du sens de celle-ci pour autant que ce dernier ne saurait être exhibé dans des lois du type de celles que visent les sciences de la nature. Mais d'un point de vue supérieur, une telle philosophie de l'histoire n'est qu'une prolégoménologie, une recherche dont la fonction est de rendre possible et de préparer une philosophie de l'histoire entendue désormais comme une histoire universelle, dont le discours repose sur celui des historiens sans toutefois le reproduire servilement, puisque le système des valeurs y est désormais l'instrument d'une herméneutique de cette histoire des historiens. L'idée rickertienne d'un système des valeurs contribue donc à la détermination d'une philosophie axiologique de l'histoire en se situant à la jonction des deux sens que celle-ci peut recevoir avec une égale légitimité.

\footnotetext{
${ }^{59}$ Cette double objection de formalisme et de gratuité, qui n'a jamais cessé d'être élevée à l'encontre de la théorie rickertienne de la science (et dont on peut dire que R. ARON fut en France le premier propagateur, dans la mesure où elle constitue rien de moins que le dernier mot de son exposé de la fondation rickertienne des sciences historiques - cf. op. cit., p. 149-155) serait ainsi également valide sur le versant de la théorie de la connaissance. Et l'on notera que Rickert semble parfois se faire à lui-même l'objection de formalisme dans l'édification du système (cf. H. RICKERT, « Le système des valeurs », trad. cit., p. 156 : «n'avons-nous pas construit un domaine auquel aucune réalité effective ne correspond, par amour des seuls principes de notre système ?»). Mais ce questionnement est à l'évidence purement rhétorique. Une réponse complète à l'objection de formalisme gratuit suppose quant à elle de montrer que l'opposition de la forme et de la matière sur laquelle elle repose relève d'un niveau que la philosophie critique des valeurs entend précisément surmonter - démonstration qui excède de loin les limites de ce travail.
} 
D'un autre côté, ce même système des valeurs unifie les deux déterminations qu'il permet de distinguer dans la mesure où, dans les deux cas, il confère à la philosophie de l'histoire une dimension critique qui vient s'ajouter à sa dimension analytique, et ce au sens fort qu'est susceptible de recevoir la notion de «critique » dans une philosophie des valeurs une dimension proprement évaluative. Disposant, avec le système des valeurs, des principes axiologiques de l'universum historique, la philosophie de l'histoire comme prolégoménologie se donne simultanément les moyens d'exercer une évaluation critique de tout discours philosophique sur l'histoire à prétention explicative totalisante. Quant à la philosophie de l'histoire en son sens éminent, nous avons vu qu'elle devait être entendue comme la description axiologique des époques passées, c'est-à-dire l'évaluation de leur sens relativement à la réalisation progressive de valeurs supra-historiques dans l'histoire humaine. Ce faisant, le système des valeurs a pour effet remarquable de pluraliser l'histoire universelle (car il y a potentiellement autant d'histoires universelles qu'il y a de «valeurs en propre ») tout en l'installant dans une dépendance relativement à l'histoire effective, qui la préserve définitivement de toute construction spéculative et lui permet de demeurer fidèle au sens proprement kantien du criticisme.

Mais il faut noter que cette fidélité s'atteste également dans un fait que nous nous bornerons à évoquer pour clore notre propos, mais qui exigerait assurément des analyses détaillées : à savoir qu'un tel refus de toute philosophie spéculative de l'histoire n'a pas pour contrepartie le renoncement à toute métaphysique. Au contraire, on peut assez aisément apercevoir une certaine exigence métaphysique à l'horizon de l'anti-historicisme de Rickert. Nous avons vu en effet que son élévation graduelle vers le concept le plus éminent de la philosophie de l'histoire dessinait simultanément le chemin d'une réfutation de plus en plus profonde du relativisme historiciste, culminant dans la neutralisation de l'opposition entre l'historique et le systématique au profit d'une complémentarité en vertu de laquelle il est montré non seulement qu'une science exhibant de façon systématique «les présupposés axiologiques de la culture $»^{60}$ est exigée, malgré son asystématicité, par l'histoire elle-même si cette dernière doit avoir un sens en général, mais aussi, à un niveau supérieur, qu' « il n’y a donc en définitive pas de sciences de l'histoire sans philosophie de l'histoire ${ }^{61}$. C'est la raison pour laquelle Rickert peut dire de sa propre critique de l'historicisme qu'elle n'est rien d'autre qu'une tentative pour «se rapprocher, au sein de l'historique même, de ce qui

\footnotetext{
${ }^{60}$ H. RICKERT, Science de la culture et science de la nature, op. cit., p. 193.

${ }^{61}$ Ibid., p. 187.
} 
transcende l'histoire $»^{62}$. Il nous semble qu'une telle déclaration peut parfaitement être comprise comme l'affirmation selon laquelle l'histoire constitue en elle-même un chemin vers la métaphysique, à condition toutefois de ne pas tomber dans la confusion, que nous avons déjà dénoncée plus haut, entre philosophie des valeurs et simple axiologie. Car, dans la philosophie de Rickert, il y a bien un lieu pour une métaphysique, mais il ne s'agit pas de la sphère transcendante des valeurs ineffectives; il s'agit bien plutôt, une fois encore, de cette sphère dans laquelle se joue l'articulation de l'être et du valoir ${ }^{63}$. Si l'on accepte avec Rickert de nommer «métaphysique» ce lieu supra-réel où se croisent et se joignent l'effectivité ontologique et l'ineffectivité axiologique, on aperçoit dès lors que l' « achèvement de tout le système philosophique ${ }^{64}$ (et non seulement de la théorie pure des valeurs), rendu possible par le système des valeurs dans la mesure où il est mis au service de l'idée d'une histoire universelle, - que cet achèvement est en lui-même l'achèvement de la réfutation de l'historicisme sous la forme d'une justification de la métaphysique depuis l'histoire et par le moyen de l'histoire.

\footnotetext{
${ }^{62}$ H. RICKERT, Science de la culture et science de la nature, op. cit., p. 192.

${ }^{63}$ Cf. Ibid., p. 188, note ; pour des développements plus précis, cf. Les problèmes de la philosophie de l'histoire, op. cit., p. 172-173.

${ }^{64}$ Ibid., p. 176.
} 